\title{
Pharmaceutical Care Network Europe Abstracts 5th Working Conference
}

\author{
21-24 February 2007
}

Published online: 7 July 2007

(C) Springer Science+Business Media B.V. 2007

\begin{abstract}
s of the short communications
Victoria Losinski, Christina Cipolle, University of

Minnesota, St Paul, USA

Realizing the transformation of a profession: the case study of the State of Minnesota
\end{abstract}

Aim of project: For over 15 years the pharmacy profession has been changing from a product to a patient centred profession with pharmaceutical care as the standard of practice. These changes have been occurring slowly, but consistently around the world. In the last year, the State of Minnesota experienced a rapid increase in the number of pharmaceutical care practices due to multiple factors. The aim of this project is to identify these factors and explore how they have contributed to changing pharmacy practice.

Method: A descriptive analysis of pharmacy practice in the United States, specifically in the State of Minnesota, was completed to identify factors which have led to increasing numbers of pharmaceutical care practices. Further analysis was completed to define how these factors aided pharmaceutical care practice development in the State of Minnesota.

Result(s): Three main factors have contributed to pharmacy's accelerated transformation: clearly defined standards of pharmaceutical care practice, accessible webbased pharmaceutical care training, and commercially available pharmaceutical care practice planning tools and resources. Minnesota legislature clearly defined the service and standards of pharmaceutical care practice for pharmacists in the State of Minnesota. This created a framework that pharmaceutical care practitioners could clearly understand and consistently demonstrate each time that they provide care to a patient. Online pharmaceutical care training was introduced to prepare pharmacists to provide pharmaceutical care using the defined standards of practice. This allowed pharmacists to access training regardless of their access to a university setting. Finally, practice development tools including a practice planning guide and documentation and billing system were made commercially available. These tools have helped pharmaceutical care practitioners create a vision for their practice and access the information they need to make it a reality. These three factors have helped Minnesota pharmacists to implement viable pharmaceutical care practices across the state and are currently being used to aid other pharmacists at a state, national, and international level resulting in a collective transformation of the pharmacy profession.

Susanne Kaae, Janine Morgall Traulsen, Birthe Søndergaard, Lotte Stig Haugbølle The Danish University of Pharmaceutical Sciences, Copenhagen, Denmark

The first Danish publicly reimbursed cognitive service in community pharmacies on a national scale - why and how did it become reality?

Aim of project: Contracts for third party reimbursement of cognitive services in community pharmacies are becoming increasingly frequent. The aim of this study is to explore why and how. The driving forces of the two main actors negotiating on the first contract of a publicly reimbursed cognitive service on a national scale in Danish community pharmacies-"Check the inhalation" will be explored. "Check the inhalation" consists of a 20 min check by the 
pharmacy staff on asthma patients' inhaler technique, when using asthma devices.

Method: An analysis of the literature describing the historic and political process leading to "Check the inhalation" will be presented. The analysis is based on two different theoretical approaches: "a) "neo-weberian" profession theory - to explain the driving forces of Danish community pharmacies and b) an approach regarding analysis of policymaking in the field of pharmacy-laid out by Traulsen/ Almarsdottir-to explain the driving forces of Danish politicians, when negotiating on "Check the inhalation".

Result(s): Since the beginning of the 1990's, Danish community pharmacies have made pharmaceutical care and provision of cognitive services a key survival strategy-as historic monopolies such as producing and dispensing medicines have been deregulated. Many within the pharmacy sector have recognized that third party payment essential in order to strengthen permanent implementation of cognitive services. Policy making in the field of medicine including pharmacy, has for the last 5 years, been dominated by a cost containment view. Danish politicians have however started recognising, that rational pharmacotherapy might be an additional solution to reduce rising costs on medicine. Danish pharmacies have been active in political work prior to "Check the inhalation", pressing forward their argument, that pharmacies could undertake tasks of rational pharmacotherapy.

Conclusion: "Check the inhalation" became a reality, as Danish politicians began recognising the economic potential, community pharmacies have in providing services of rational pharmacotherapy. Danish community pharmacies have been politically active in this process, as third party payment of cognitive services to them, is important as a survival strategy.

I. Krass ${ }^{1}$, C. Delaney ${ }^{2}$, S. Glaubitz ${ }^{3}$, T. Kanjanarach ${ }^{1}$. (1) Faculty of Pharmacy, Sydney, Australia. (2) St Vincent's Hospital Sydney, Australia. (3) University of Utrecht, the Netherlands

\section{Patient satisfaction with Pharmacy Diabetes Disease State} Management (DSM) Services: validation of an instrument

Aim of project: To develop and validate an instrument to measure patient satisfaction with Diabetes Disease State Management services delivered by community pharmacists.

Method: The Diabetes Disease State Management Questionnaire (DDSM-Q) was developed on the basis of 14 in-depth, semi-structured interviews with participants, in the Pharmacy Diabetes Care Program in New South Wales ${ }^{1}$. The results of the qualitative interviews informed development of a 32 item questionnaire. The DDSM-Q was next sent to 165 participants of the pharmacy diabetes care projects. Principal Components Analysis (PCA) followed by confirmatory factor analysis (CFA) were used to assess construct validity and Cronbach's alpha for internal consistency. Concurrent validity was examined by correlation between the factor scores and change in $\mathrm{HbA}_{1 \mathrm{~g}}$ levels.

Result(s): One hundred and seventeen questionnaires were returned, a $71.3 \%$ response rate. Respondents had a mean age of 63 years and $60 \%$ were male. PCA identified 4 factors (17 items) contributing to consumer satisfaction and this was confirmed with CFA (model. fit: X"191 .68; df $=115$; bootstrap $P=0.130$ ). These were labelled "impact on diabetes self management", "satisfaction with improvement in knowledge" and "affect based satisfaction with the pharmacist's service" and "performance of the pharmacist's service" with Cronbach's alphas of 0.83 , $0.80,0.62$ and 0.77 , respectively. There was a statistically significant correlation between satisfaction scores and changes in $\mathrm{HbA}_{1 \mathrm{~g}}$.

Conclusion: The results support the validity and reliability of the DDSM-Q as a measure of patient satisfaction with pharmacist-delivered DSM services for type 2 diabetes. Further research will be needed to validate the instrument in different populations.

1. Krass et al. The Pharmacy Diabetes Care Program. http://lwww.guild.org.au/uublic/researchdocs/2OO2518 fr.pdf, last accessed 8/12/06. (Funded by CDHA under the 3rd Community Pharmacy Agreement).

Mrs. Mirjam E.A.P. Kokenberg, Quality Institute for Pharmaceutical Care, Kampen, The Netherlands

Best practice polypharmacy: the road from a single project to a nationwide best practice

Aim of project: The development of a nationwide implementation of polypharmacy intervention which consists of optimising pharmacotherapy of an individual multi-druguser, by medication review by physician and pharmacist together.

Method:

- In 1999, 2000 and 2001 QIPC studied the organization, feasibility the medication changes and the financial outcomes of polypharmacy intervention in three pharmacies.

- In 2004 a major healthcare insurance company showed interest in the polypharmacy intervention and financed an implementation study in 14 pharmacies and 31 physicians, performed by QIPC.

- In 2005 the Dutch Ministry of Health nominated the QIPC polypharmacy intervention (together with the 
$\mathrm{DGV}^{*}$ polypharmacy intervention) as 'Best Practice'. QIPC and DGV developed a joint Best Practice Polypharmacy structure. A nationwide web based data system was developed for registration of the interventions on patient level.

- In 2006 the Dutch Ministry of Health partially subsidized the nationwide implementation of this joint Best Practice Polypharmacy project by QIPC and DGV. This Best Practice consists of:

- Selection of patients

- Medication review by pharmacist and physician separately

- Joint consultations between pharmacist and physician

- Evaluation on patient level

- Monitoring and evaluation of the interventions

- Continuity of the interventions

- The pharmacists and physicians are coached by DGV and QIPC for 9 months.

- In 2007 the implementation will continue.

Result(s):

- The published studies in 1999, 2000 and 2001 showed that the polypharmacy interventions are feasible and worthwhile. DRP's were found and solved, the costs of medication decreased.

- The study of 2004 (839 patients) shows that in 36\% of the reviewed patients a medication change is necessary; stopping medication happens most often (44\%), followed by change in doses $(23 \%)$, change of medication $(20 \%)$ and starting new medication (13\%). 35\% of the patients says to feel better after the intervention, $97 \%$ of the patients thinks a periodically performed medication review very important, $36 \%$ of the pharmacists and physicians thinks their professional working relation improved.

- In 200643 groups of pharmacists and physicians are now implementing the 'Best Practice Polypharmacy', and preliminary data (315 patients) show that in about $50 \%$ of the patients a medication change is necessary.

- In 2007 QIPC and DGV aim at coaching 30 groups intensively and 200 groups less intensively.

Conclusion: (1) Because of the cooperation between physician and pharmacist outcomes are achieved which neither of them could achieve individually. (2) Ready and easy to use materials, ICT and coaching are necessary to get pharmacists and physicians starting. (3) Practice is creative: the intervention still develops while optimal health care is provided.

*DGV stands for the Dutch Institute for safe use of medicines
Veerle Foulon ${ }^{1}$, S. Sirmoens ${ }^{1}$, G. Laekeman ${ }^{1}$ and P. de Witte $^{2}$. (1) Research Centre for Pharmaceutical Care and Pharmaco-economics, K.U.Leuven, Belgium. (2) Programme Director, Faculty of Pharmaceutical Sciences, K.U.Leuven, Belgium

A conceptual framework for the implementation of pharmacy practice research in the education of students at the K.U.Leuven, Belgium

Aim of project: In consequence of the Bologna declaration of 1999 and the following reform of the Flemish system of higher education, a master thesis will be one of the requirements to obtain the degree of Master in Pharmaceutical Care from 2007-2008 onwards. The aim of this project was to develop a conceptual framework for a master thesis in pharmaceutical care, along with the design of an adapted curriculum.

Method: On the basis of a review of recent literature and an analysis of study programmes of different

European faculties of pharmaceutical sciences, a core group of staff members formulated objectives for the Master in Pharmaceutical Care. These objectives, considering knowledge as well as attitudes and practices, were discussed in focus groups with community pharmacists, hospital pharmacists and students. The resulting competency profiles were approved by the faculty council and faculty members agreed upon the particular competencies that should be attained in the different courses. For the master thesis, a concept was worked out that integrates different aspects of the existing study programme and that gives students the opportunity to develop research skills. The major parts are 1) literature review; 2) project work; 3) pharmacy practice research and 4) writing up the findings.

Results: The master thesis will be organized for groups of 6 students, working on a central theme. Every division of the faculty will supervise one group of students, hence stimulating the integration of fundamental research and pharmacy practice. Making an individual review of the literature, addressing one particular issue of the theme, will prepare students for discussions and help them to develop research questions. Project work will bring them in contact with a professional organization or a pharmaceutical company. The pharmacy practice research project will be conducted along with the internship in a community pharmacy. We strongly believe that this will convince both students and supervisors of the value of the discipline and accelerate the implementation of pharmacy practice research in Flanders. Finally, writing a dissertation in which the findings are confronted with the literature data will undoubtedly contribute to the education of critical pharmacists, aspiring for a greater role in health care. 
Divaldo Pereira de Lyra Jr., Fabiola Sulpino Vieira, Manoel Roberto da Cuz Santos, Federal University of Sergipe, Aracuja, Brazil

\section{Introducing actions to implement Pharmaceutical Care research in Brazil}

Aim of project: To introduce actions to implement Pharmaceutical Care research in public health care system in Brazil

Method: In August 2005, Pharmaceutical Assistance Department (DAF) of Brazilian Health Ministry introduced National action plan to implement and develop Pharmaceutical Care research in public health care system (SUS). This plan was designed because although there were no studies about drug-related morbimortality in Brazilian population, drugs have been identified as the first cause of intoxication in this country. Thus, the DAF, established a financial funding to support research in SUS, including Pharmaceutical Care. After this, three events were organized and financed by DAF to promote interchange between Brazilian and foreigner researchers.

Result(s): Of the 27 proposals submitted to Brazilian Scientific Commitee, only 11 projects received financial support of DAF. Nowadays, these projects are being developed in different settings (hospitals, ambulatories and community pharmacies) of all Brazilian regions. In March 2006, DAF organized the 1st National Forum of Pharmaceutical Care research in SUS with 40 Brazilian researchers to present the projects approved and to discuss the importance of these studies to promote the rational medication use in Brazil. In May 2006, nearly 120 experience reports of 16 Brazilian states were submitted to Scientific Committee of the 1st International Seminar of Pharmaceutical Care research in SUS and these reports, 90 were approved to participate. The researchers had opportunity to discuss with speakers of Spain, USA, Cuba and Argentina. Besides, they participated of workshops with pharmacist practioners and managers of Public Health care system formulating strategies to implement Pharmaceutical Care research in SUS. In September 2006 occurred the 1st Brazilian Symposium of Clinical Pharmacy with presence of speakers of Australia, New Zealand and Japan. In this event was discussed the importance of post-graduation in formation of Pharmaceutical Care researchers and practioners. Based on the results of these events, DAF jointed a Group with goal of training to Brazilian pharmacists to practicing the pharmaceutical care, as well as, to create a net of researchers to monitor and interchange the knowledge obtained by the projects supported by DAF.

\section{Abstracts of the posters}

H. Oller Dolcet, S.I. Benrimoj, F. Fernandez-Llimo. Faculty of pharmacy, Sydney, Australia

A theoretical framework for the implementation of patientoriented pharmacy services using a quality management system

Aim of project: The aim is to develop a sustainable quality management system based on the concept of continuous quality improvement grounded in a rigorous theoretical framework.

Method: A literature review comprising of a search of the following journals: "International Journal for Quality in Health Care", "Joint Commission Journal of Quality Improvement", "Quality in Health Care" (1980-2005) and the libraries databases of the University of Sydney and Barcelona using the terms Quality assurance, Quality improvement, Quality service, Quality management and Health Care was undertaken.

Results: Six theoretical frameworks were brought together to develop a conceptual approach. These frameworks included the "Plan-Do-Check and Act" cycle (Shewart, 1920's), the "14 Principles" (Deming, 1930's), the "Juran Trilogy" (Juran, 1940's), the "Quality Control Cycle" (Ishikawa, 1940's), the "Structure-Process-Outcome" model (Donabedian, 1960's) and the "Quality Management Model" (Crosby, 1980's). The proposed model comprises of four main stages, with particular emphasis on practical implementation and the process of long term sustainability. All stages are cyclically interrelated as per the concept of continuous quality improvement.

The system background is an enabling stage where the required infrastructure is assembled. The system design is the analysis of both the internal systems and the services provided as per the international quality standards (ISO, EFQM \& JCI). The third stage is the system implementation, through the establishment of a quality centre. The system outcomes are evaluated using behavioural changes, health care and business impact indicators. Simulated patient methodology will be used as a surrogate measure to assess the outcomes as well as implementation criteria. As the framework has been developed using continuous quality improvement theory, it is inherently cyclic in nature. The system can constantly be improved and it will be the basis for implementing patient-oriented pharmacy services. The empirical research will be conducted with a group of Spanish community pharmacies. 
Costa F.A. ${ }^{1}$, Guerreiro M.P., Hughes C., McElnay J.C. (1) Institute of Health Sciences Egas Moniz, Portugal

\section{Applying the Behavioural Pharmaceutical Care Scale (BPCS) in Portugal}

Aim of project: To evaluate the degree of implementation of pharmaceutical care in Portugal.

Method: A cross-sectional survey was used to gather information on the type of services routinely provided by Portuguese community pharmacists (01-03/2006). Data were collected through a linguistically adapted and piloted version of the BPCS for Portugal ${ }^{1,2}$, comprised of two sections. Section A characterized the pharmacy premises, staff and general services. Section B evaluated services provided to the last patients entering the pharmacy, referring to a specific time period, using 34 vignettes grouped in three domains: Direct Patient Care Activities (DPCA; 17 items; score range $=0-85$ ), Referral and Consultation Activities (RCA; 8 items; score range $=8-40$ ) and Instrumental Activities (IA; 7 items; score range $=7-35$ ). These are added up to form the BPCS score (range $=15-160$ ). All Portuguese pharmacies received the adapted questionnaire $(n=2805)$, aiming for $n=561 \quad(P=21 \% ; \mathrm{d}=3 \%$; 95\%CI $)$. Data were analyzed for descriptive statistics using SPSS v.13.0.

Results: A total of 564 pharmacists returned the questionnaire $(20.1 \%)$. Within the DPCA Dimension, documentation activities were rated low, whilst those involving direct contact with the patient were particularly strong $($ DPCA $=30.6 \pm 13.9)$. Within the RCA Dimension, the worst rated activities involved direct communication with the GP $(\mathrm{RCA}=19.8 \pm 5.5)$. The highest scoring activities in the IA Dimension were the provision of medical information to patients, the double-validation of prescriptions, and the use of a private counseling area. The lowest scoring was assessment of patient satisfaction and performance evaluation $(\mathrm{IA}=23.8 \pm 3.7)$. The BPCS overall score in Portugal was 71.13.

Discussion: Portuguese BPCS score was slightly lower than those reported for Northern Ireland $(=74.1)$ and Switzerland (=76.3), suggesting further improvement in the implementation and dissemination of pharmaceutical care in Europe.

(1) Odedina \& Segal. Behavioral pharmaceutical care scale for measuring pharmacists' activities. Am J Health-Syst Pharm 1996;53:855-65.

(2) Bell, McElnay, Hughes, Woods A. Provision of pharmaceutical care by community pharmacists in Northern Ireland. Am J Health-Syst Pharm 1998;55:2009-13.
C. Rossing (crapharmakon.dk), H. Herborg, L. Sorensen, Pharmakon, Hillerød, Denmark

\section{Adherence problems among type 2-diabetics}

Aim of Project: To generate a sample of type 2-diabetics with a risk of adherence problems for a controlled intervention study. To estimate the frequency of adherence problems among type 2-diabetics who were willing to participate in such a project aimed at exploring and improving safe and effective use of medicines in a Danish community pharmacy setting.

Method: From the Danish county of Funen, 6 pharmacies were included in the project. Potential project patients were identified from pharmacy records as patients who were aged between 18 and 80 years and had reimbursed prescriptions of anti-diabetics in the period from 01.01.2006 to 30.09.2006. Altogether 2,017 patients were informed by mail about the project. Patients were included in the project if they administered their medication themselves, gave written consent to participation and returned a completed questionnaire on medication use, medication behaviour plus some clinical measures.

Eight criteria were used to categorize patients as having a risk of adherence problems including: reported elevated HbAlc (above 6.1\%) and/or elevated blood pressure (above $130,180 \mathrm{mmHg}$ ) concurrent with the GP's dissatisfaction with the value of these clinical measures; deviations in medication taking behaviour within the previous 2 weeks; dissatisfaction with the effect of their medicines; experienced problems with their medicines, and poly-pharmacy.

Result(s) and conclusion(s): In total, 315 patients consented to be included in the study. The mean age was 63.3 years, and $191(60.6 \%)$ were males. According to the criteria, 205 responders $(65.1 \%)$ were categorized as having a risk of adherence problems. The most frequent problems were elevated clinical values $(23.1 \%)$, deviation in medication behaviour (37.8\%) and polypharmacy $(15.8 \%)$ as risk-factors of non-adherence.

Marleen Haems, Kava, Antwerp, Belgium

\section{KAVA-Foldermanagement: a contribution for written patient information of good quality}

Aim of project: Patients are empowered by being informed about their diseases and about their medicines. On the legal side the European directive demands that every medicine is accompanied by a patient insert. These patient leaflets have to meet legal criteria. On the other side many patient folders about pathologies, drugs, health, ... are developed from different origin: insurance companies, pharmaceutical 
industry, health authorities, universities, professionals associations etc.

As a consequence the health care provider (doctor, pharmacist, ...) can dispose over a very large amount of written patient information. The quality however is not always as one should expect and patient leaflets used in daily practice are sometimes of a rather low quality. With the project "Folder management" we try to offer a tool for selection of adequate patient information

Method: Leaflets are collected by the Folder management team and the technical readability is evaluated according to the criteria of Flesh-Douma. Secondly, correctness of content, lay out, and usability in daily practice is evaluated according to determined criteria.

The leaflets which respond positively to the evaluation procedure are communicated to the profession by publication and by mentioning on a special webpage. Leaflets which do not respond to the criteria are not mentioned. Evaluation criteria are communicated to third parties, so that they can be taken into account when new patient material is made.

Result(s): 54 patient leaflets are evaluated so far. 21 passed the test. Written patient information is necessary, especially in the pharmaceutical care context. However, the majority of available patient leaflets does not meet the criteria of technical readability, content correctness, layout and usability as developed in this project. Examining leaflets thoroughly before using them in daily practice is necessary. But time is very precious in daily pharmacy practice and little time is spent on the selection and evaluation of the patient material that will be used. By consulting the results of the Project Folder management the pharmacist can easily select appropriate and qualitative patient leaflets for specific indications without spending too much time. With our project "folder management" we hope to offer a service that contributes to a better quality of written patient information

\section{Lastra CF, Modamio P, Sebarroja L, Casasin T, Marifio} EL. Clinical Pharmacy and Pharmacotherapy Unit. Faculty of Pharmacy. University of Barcelona. Avda. Joan XX11l sin. Barcelona (Spain)

\section{EDEMED: a computer tool about Medication Errors and drug safety for implementation of pharmaceutical care}

Aim of project: Medication Errors (ME) are one of the major aspects that impacts the practice of pharmaceutical care, currently resulting in the development of actions directed to their prevention. The objective of the present communication is to show the results of the notification of ME carried out by pharmacy students from an interactive program, developed according to the recommendations of the Bologna Declaration and of the European Higher Education Space (EHES).

Method: The interactive program that has been designed is called EDEMED: Errors of Medication, financed by the Agencia de Gesti6 d' Ajuts Universitaris i de Recerca (AGAUR) of the Generalitat of Catalonia, which can be accessed at the following web site: http://www.ub.edulfarmaciaclinicalprojectes/errors/.

The program is structured in two blocks. The first block is a self-learning procedure module composed of eight sections through which the student sequentially deepens the topics of the security of the medications. The second is a voluntary notification system of ME whose objective is to enable the student to detect possible ME among his or her relatives, friends, work, etc. Students can introduce this information in the program, generating a database that be introduced in the Program of Prevention of Medication Errors of Catalonia.

Result(s): One hundred and eleven students voluntarily participated during the second semester of 2006. The total number of notifications carried out thus far is 131, of which, after their analysis and evaluation, 122 were accepted, averaging one ME per participating student (maximum of 6-minimum of 1). Of the errors notified, $76 \mathrm{did}$ not arrive at the patient (62.3\%). They were detected in their greater part in community pharmacy $(67.2 \%)$ and in primary care health centres $(13.1 \%)$. The main causes were prescription errors (32.8\%) and validation/dispensing errors $(15.1 \%)$.

Reviewing the results, it can be stated that the utilization of this educational tool, which is directly related to the professional pharmaceutical practice, can clearly contribute to an integration of theory and initiation of investigation, especially in enhancing the security of patient use of medications according to the EHES.

Feletto E, Oller Dolcet H, Femandez-Llirnos F, Roberts A, Benrimoj SI, Faculty of Pharmacy, Sydney, Australia

An international approach to linking quality systems and business capabilities to enhance viability and the implementation of patient orientated services in community pharmacy

Aim of project: The aim of this research is to develop sustainable systems for community pharmacy that are applicable globally across various health care systems, cultures and pharmaceutical environments. This may include quality and capabilities systems that facilitate the implementation of patient orientated services. This research is being conducted on an international level to 
overcome the current limitations and reduce the homocentric nature of research results.

Method: The research parallels the development of two research projects-one being conducted in Spain ${ }^{1}$ and the other in Australia $^{2}$. Two interdependent literature reviews were merged. One review focused on continuous quality improvement in community pharmacy and comprised a literature search in: "International Journal for Quality in Health Care", "Joint Commission Journal of Quality Improvement" and "Quality in Health Care" journals from 1980-2005. Search terms such as quality assurance, quality improvement, quality service, quality management and health care were used. The other search analysed literature from the Medline, IPA, Embase, AB1 Inform and Business Source Premier databases. Terms such as pharmacy, management, business model, pharmacy services and business viability were used, with no date limits applied to the search.

Result(s): The resulting figure that will be presented shows the merger of these two concepts and highlights business viability as being the overall outcome for community pharmacy. This ensures that the primary aim of community pharmacy remains focused on high quality pharmaceutical care for the population and patients. Business viability is not determined solely by financial measures but as a composite of business impact and health care impact. The combination of these two factors can provide metrics on a number of levels which could be indicative of the degree of that pharmacy's system sophistication.

References

1. Oller Dolcet H. Fernander-Llimos F. Benrimoj SI (2006) A theoretical framework for the implementation of patient orientated pharmaceutical services using a quality management system. PCNE WC 2007 Abstract Poster 1.

2. Feletto, E. Roberts, Benrimoj SI (2006) Optimising business viability in Australian community pharmacies by integrating cognitive pharmacy services. PCNE WC 2007 Abstract Poster 13.

Feletto E, Dunphy D, Palmer I, Benrimoj SI, Roberts A. Faculty of Pharmacy, Sydney, Australia

\section{An industry approach to practice change in community pharmacy}

Aim of Project: Advancing the professional role of community pharmacy is an international trend that is proving to be challenging for the industry. Effective change should be implemented at multiple levels of a profession, from individual pharmacy to industry level. Identifying new opportunities and understanding how to roll out new programs are important elements of multifaceted change. Research was lacking on strategies for industry change in community pharmacy, as the focus had been on individual pharmacies. The absence of a systematic approach at this higher level may be impeding change for individual pharmacies. The aim, therefore, was to provide a structure and system for industry change to occur.

Method: Two concurrent studies were conducted to achieve these aims. One identified trends through an extensive literature review relating to future trends in health. The literature was divided into the following categories: future certainties, opportunities for pharmacy, consequences for pharmacy, and consequences for others. Then areas of particular interest were identified, classified and assessed and the implications for pharmacists and their relationship with other health care professionals were outlined. The second part involved consumer forums held with 68 consumer representatives and 51 semi-structured interviews with key pharmacy stakeholders.

Result(s): The findings of the first study resulted in the development of the Characterising Opportunities Filter (COF). It is a systematic approach to selecting cognitive pharmacy services in the context of future health care. Specifically, COF characterizes the opportunities for community pharmacy in seven dimensions. The developed Pharmlnd Wheel presents issues relevant to designing and implementing a change program for the industry as a whole. Industry level change is imperative because if changes are not introduced across the whole industry, the industry may act as a brake on attempts by pharmacists to make changes to service delivery in their pharmacies.

Feletto E, Dunphy D, Palmer I, Benrimoj SI, Roberts A, Faculty of Pharmacy, Sydney, Australia

\section{Practice change in community pharmacy—tools for service Implementation}

Aim of Project: Practitioners, policy makers and academics alike have recognized the need for well developed implementation processes when launching new pharmacy initiatives. The change management discipline can provide a systematic approach to introducing cognitive pharmacy service in community pharmacies. The "Change Management and Community Pharmacy" research project was designed to address some of the issues that arise in change management in Australia. One of the main project aims was to develop practical change tools to support community pharmacies for service implementation.

Method: The results came from a synthesis of information gathered from Australian pharmacies during the 12 month project. The methodologies used include: 
- 10 focus groups $(n=102)$

- 24 case studies of community pharmacies

- a quantitative mail survey with 2,022 community pharmacy owners, response rate of $\mathbf{2 8 . 5 \%}(n=575)$

Data were analysed using a systematic social science content analysis method.

Result(s): Three tools for pharmacy practitioners were developed: the Pharmacy Viability Matrix, the Pharmacy Change Readiness Wheel and the Pharmacy Change Implementation Wheel. The Pharmacy Viability Matrix presents four different strategic business models for pharmacy that are defined by their range of products and their community scope. The Wheels were developed to help practitioners practically implement changes. The Pharmacy Change Readiness Wheel is an assessment tool that quantifies the pharmacy's preparedness for change. Then, the Pharmacy Change Implementation Wheel presents a systematic approach to planning and implementing a change program for service.

Feletto. E, Roberts, A \& Benrimoj SI. Faculty of Pharmacy, Sydney. Australia

\section{Optimising business viability in Australian community pharmacies by integrating cognitive pharmacy services}

Aim of project: Integrating cognitive pharmacy services (CPSs), under the umbrella of pharmaceutical care, into existing pharmacy business models is challenging. Previous research conducted by Roberts et al, shows that implementation can be accelerated through a number of practice change facilitators. In Australia remuneration has been achieved for some selected CPSs but, alone, does not guarantee service implementation and the viability of the community pharmacy. As the role of pharmacists develops so too should the business models of pharmacy. The aim of this project is to develop a conceptual model to guide research on how Australian community pharmacies optimize their business viability within the changing external environment.

Method: A literature review was conducted, using the Medline. IPA, Embase, AB1 Inform and Business Source Premier databases. Terms such as pharmacy, management, business model, pharmacy services and business viability were used, with no date limits set for the search. The conceptual model presented below shows the interdependent relationships developed from looking within and outside the pharmacy literature.

Resul $t(s)$ : The framework developed shows that systemized management processes could potentially be used to help pharmacists provide these cognitive pharmacy services whilst optimising business viability. This includes highlighting business flexibility capabilities. Additionally, systemized management processes would be identified to achieve a higher level of service orientation through a sustainable business model. The research planned for this project will highlight the interdependency of health care and business impact for pharmacy and how they can be used to support the implementation of cognitive pharmacy services. Practical applications for pharmacy practitioners will also be developed.

Catriona Bradley ${ }^{1,2}$, Martin Hemman ${ }^{1}$. (1) School of pharmacy, Trinity College Dublin, Ireland. (2) Boots the Chemist, Ireland

Studies of health promotion and weight control in Community Pharmacy in the Republic of Ireland

Aim of project: To investigate attitudes and practices of community pharmacists in the provision of health promotion advice.

Method: Three studies explored health promotion within community pharmacy.

Five focus groups investigated pharmacist attitudes to health promotion, and identified factors which facilitate or hinder the provision of health promotion advice. Themes and sub themes were identified using NVivo Version 2.0. In a simulated patient study, researcher (BMI $22 \mathrm{~kg} / \mathrm{m}^{2}$ presented at 20 randomly selected urban pharmacies, seeking advice on weight control. Responses were recorded on a structured feedback form.

A pilot weight-control programme, enabled pharmacists to provide accurate, patient-centred information on weight control in a time-efficient way. This was delivered to twenty overweight and obese patients over 3 months. Before and after measurements of weight, BMI and waist circumference were used as outcome measures.

Results: From the focus groups the main factor identified as facilitating the delivery of health promotion was the positive nature of the pharmacist-patient interaction. Barriers included difficult relationship with other health professionals, lack of finance, lack of time and lack of expertize. Pharmacists expressed a lack of confidence in organising structured interventions and in providing advice on sensitive topics, such as weight loss.

The simulated patient study found that the most common questions asked were about desired weight loss (7/20) and the period of time over which the weight was to be lost $(6 / 20)$. None of the pharmacy personnel asked why the researcher wished to lose weight and none of them measured the researcher's BMI. The health promotion information provided was general, with little effort made to tailor the advice for the patient. 
Table 1 Frequencies of single activities and scores of the original dimensions

\begin{tabular}{|c|c|c|c|c|c|c|c|}
\hline & \multicolumn{4}{|c|}{$\begin{array}{l}\text { When delivering a new prescription for chronic conditions pharmacist reported (freq. } \\
\text { Of activity in \%) }\end{array}$} & \multicolumn{3}{|c|}{$\begin{array}{l}\text { Mean scores } \pm \text { SD and reliability estimates } \\
(\text { Cronbach's } \alpha) \text { range } 1-5(1=\text { never } 5=\text { always }\end{array}$} \\
\hline & $\begin{array}{l}\text { to document } \\
\text { therapeutic } \\
\text { objectives }\end{array}$ & $\begin{array}{l}\text { to question what patients } \\
\text { hope to achieve from drug } \\
\text { therapy }\end{array}$ & $\begin{array}{l}\text { to document the } \\
\text { patient's medical } \\
\text { condition }\end{array}$ & $\begin{array}{l}\text { to check for } \\
\text { drug-related } \\
\text { problems }\end{array}$ & $\begin{array}{l}\text { Direct patient } \\
\text { care activities }\end{array}$ & $\begin{array}{l}\text { Referral and } \\
\text { consultation } \\
\text { activities }\end{array}$ & $\begin{array}{l}\text { Instrumental } \\
\text { activities }\end{array}$ \\
\hline $\mathrm{CH}$ & $4.7 \%$ & $15.8 \%$ & $20.8 \%$ & $85.2 \%$ & $2.2 \pm 0.70 .797$ & $2.2 \pm 0.60 .740$ & $2.9 \pm 0.80 .67$ \\
\hline BW & $6.6 \%$ & $25.9 \%$ & $16.2 \%$ & $83.0 \%$ & $1.9 \pm 0.90 .884$ & $2.5 \pm 0.70 .755$ & $2.7 \pm 0.80 .706$ \\
\hline SA & $7.7 \%$ & $23.1 \%$ & $16.3 \%$ & $95.5 \%$ & $2.1 \pm 0.90 .854$ & $2.6 \pm 0.70 .770$ & $2.7 \pm 0.80 .684$ \\
\hline
\end{tabular}

The three regions show rather similar results on aggregated activity levels but more differences if one goes into detailed questions. It can be assumed that the latter provide more insight into differences of the practice of pharmaceutical care in both countries. At the same time they give important information about how to improve special activities of pharmaceutical care.

Twenty patients were recruited to the weight control programme and 5 dropped out. After three months, statistically significant reductions in weight $(-3.55 \mathrm{~kg})$, BMI $\left(-1.36 \mathrm{~kg} / \mathrm{m}^{2}\right)$, waist circumference $(-6.29 \mathrm{~cm})$ and percentage weight loss $(4.15 \%)(P<0.002)$ were achieved. This work' provides good insights into the current health promotional role of community pharmacists and will inform the development of larger scale studies.

Patrick Eichenberger ${ }^{1}$, Marion Schaefer ${ }^{2}$, Kurt E. Hersberger ${ }^{1}$. (1) Institute of Clinical Pharmacy. University of Basel, Switzerland; (2) lnstitute of Clinical Pharmacology, Charité, Berlln, Germany

\section{Provision of pharmaceutical care by Swiss and German community pharmacists}

Aim of project: PCNE initiated a research study across European countries with the aim to examine and to compare the extent to which pharmaceutical care is being implemented into routine practice within community pharmacies. We present preliminary results from Switzerland and Germany.

Methods: Postal survey of a German version of the validated BPCS questionnaire among all pharmacies in the German speaking part of Switzerland and in 2 different regions of Germany (Baden-Württemberg, Saxony-Anhalt).

The 64-item questionnaire assessed characteristics of responding pharmacists and of their pharmacies, in tree dimensions of pharmaceutical care: Direct patient care activities, referral and consultation activities and instrumental activities (informational support or performance evaluation).

Response rates of addressed pharmacies were in Switzerland $(\mathrm{CH}) 48.2 \%$ (392 out of 814) in Baden-Württemberg (BW) 9.7\% (270/2780) and in Saxony-Anhalt (SA) $36.5 \%(222 / 608)$.
Most pharmacies (CH: 77.0\%, BW: 75.9\%, SA: 86.5\%) have a private consultation area and most of them use a drug surveillance system to assess prescribed medicines in a drug history (CH: 99.7\%, BW: 100.0\%, SA: 99.5\%), to check for potential drug interactions $(\mathrm{CH}: 97.4 \%$, BW: $100.0 \%$, SA: $100.0 \%)$, to document patient health characteristics $(\mathrm{CH}$ : $83.8 \%$, BW: $95.2 \%$, SA: $98.2 \%$ ) and to check for contraindications (CH: 50.5\%, BW: 90,7\%, SA: 90.4\%) Table 1.

Maguire $\mathrm{LK}^{1}$, Hunter $\mathrm{TA}^{2}$, Hughes $\mathrm{CM}^{1}$, Henman $\mathrm{MC}^{3}$ (1) School of Pharmacy, Queen's University, Belfast, N. Ireland. (2) CAWT Development Centre, Gransha Park, Derry.N. Ireland. (3) School of Pharmacy, Trinity College Dublin, Dublin, Ireland

Co-operation and working together (CAWT) campaign to promote awareness of type 2 diabetes

Aim of project: To improve public awareness of type 2 diabetes in Pharmacies in Northern Ireland (NI) and the Republic of Ireland (ROI)

Method(s): The campaign ran in the CAWT areas in NI and ROI during October/November 2005. All Pharmacies were included and participants attended training. Pharmacies received posters, window displays and leaflets. Pharmacists recorded queries about type 2 diabetes in the week before (no display); during (materials displayed in pharmacies relating to type 2 diabetes), and after the campaign (no display). Queries documented included; nature of -query, demographic information, existing or no diagnosis of diabetes and the phamacist's intervention. A follow-up Pharmacist Satisfaction Survey was administered 10 weeks post campaign.

Results: Eighty-six community pharmacies in the CAWT area participated in the project (51\% NI/49\% ROI) and 1192 queries were generated throughout the study: 121 $(10 \%)$ in the pre-campaign period, $894(75 \%)$ during campaign and $163(15 \%)$ afterwards. Patient-generated and pharmacist generated-queries increased by $52 \%$ and $86 \%$ 
during the campaign. $43 \%$ of patient queries came from diagnosed diabetics, $37 \%$ from patients without diagnosed diabetes and $20 \%$ from a family member. Of the pharmacist-initiated queries, $58 \%$ were with diagnosed diabetics, $27 \%$ were with patients without diagnosed diabetes and $15 \%$ were with family members. Pharmacists were more likely to generate a query with a diagnosed diabetic $(P=0.00)$. The most common query, from both the patient and pharmacist perspective related to diet ( $21 \%$ of total). A few pharmacists (18.8\%) reported knowing of at least one person diagnosed with type 2 Diabetes as a direct result of the campaign.

Vanhecke $S^{1}$, De Wulf $I^{2}$, Nagels $F^{2}$ and Sarre $S^{1}$. (1) Department of Pharmaceutical Chemistry, Drug Analysis and Drug Information, Vrije Universiteit Brussel, Belgium. (2) Centrum Wetenschappelijke Ontwikkeling voor Apothekers, APB, Belgium

\section{Effect of a centre of excellence for migraine on} Pharmaceutical Care services in a Primary care setting: a pilot study

Aim of project: In Belgium, a Centre of Excellence (CE) was created to help pharmacists gain expertize in one or a few specific disorders. The first $\mathrm{CE}$ was established for migraine in 2006. The centre offers support to pharmacists willing to provide Pharmaceutical care services for migraine patients.
Method: The aim of this pilot study was to evaluate the feasibility and the impact of the CE in a community pharmacy setting. During 3 months, several intervention tools were evaluated. Pharmacists were asked to evaluate the medication profile of five patients. This information was obtained upon appointment as well as during each visit to the pharmacy. The quality of life of the patients was estimated via standardized questionnaires [EQ-5D and the Migraine Disability Assessment (MIDAS)-test] at the beginning and at the end of the study. Participating pharmacists and patients were asked their opinion on the $C E$ via a questionnaire at the end of the study.

Result(s): Twelve pharmacists and 36 migraine patients participated in the pilot study. The patients appreciated the extra guidance and were willing to visit upon appointment. Comparing the medication profile at the beginning and at the end of the study showed a decrease in the use of analgetics and NSAIDs as well as an increase in the use of gastroprokinetics and triptans. These changes were not statistically significant. However, the use of antiepileptic drugs increased significantly. Five new patients were started on antiepileptic drugs, 2 of which were referred by the pharmacist to the general practitioner. The average MIDAS-score (which scores the intensity of a migraine attack) decreased significantly whereas the average EuroQol score increased significantly. A follow-up study including a control group is necessary to confirm the positive effects of this first $\mathrm{CE}$ on the outcome of migraine patients. 